\title{
Anti-collision systems for robotic applications based on laser Time-Of-Flight sensors
}

\author{
Luca Bascetta, Gianantonio Magnani, Paolo Rocco, Roberto Migliorini and Marco Pelagatti
}

\begin{abstract}
This paper discusses methods to avoid collisions between a robot and an obstacle, based on distance feedback given by a laser Time Of Flight (TOF) sensor. Two solutions are presented: the former (GCT: Geometry Consistent Trajectory) preserves the geometrical properties of the trajectory, while the latter (TCT: Time Consistent Trajectory) aims at preserving the time properties of the trajectory. Both the methods are validated on an experimental setup based on a two-head linear motor equipped with a commercial laser TOF sensor. Extension to a planar two d.o.f. manipulator equipped with a multiple beam sensor at the end effector is discussed as well.
\end{abstract}

\section{INTRODUCTION}

Physical human-robot interaction [1] is one of the key challenges for robotics in the next few years. A direct, physical interaction between robots and humans is essential in all those situations where a step beyond the traditional use of robotic arms is desired. These scenarios include both industrial robotics and service robotics, where the diverse applications are generally intimately based on this physical interaction (e.g. rehabilitation robots and co-workers). A key to success for the diffusion of industrial robotic manipulators in small companies is the possibility for the human operators and the robots to at least share the same workspace at the same time, while working on different tasks (coexistence). In a more ambitious perspective, the human and the robot coexist in the same workspace while working on the same task (cooperation). Besides a radical shift of paradigm in the design of the overall robotic applications, this new scenario entails basic problems of safety.

Safety in the interaction can be, to some extent, gained in a passive way, i.e. with inherently safe lightweight robots, built with mechanical solutions intended to minimize the danger potential of impacts [2]. Consequences of impacts between robots and humans are discussed in e.g. [3], [4], [5]. Alternatively, safety can be gained with an active approach, endowing robotic manipulators with additional sensors that are able to sense the position of the robot relative to obstacles (including humans), and using the resulting information in suitably designed control systems. Significant contributions in the field include [6], [7], [8]. Comprehensive overviews of physical human robot interaction issues are given in [1], [9], [10].

Active physical human-robot interaction control is generally performed through exteroceptive sensors mounted above or near the robot, or on the robot itself.

L. Bascetta, G. Magnani, P. Rocco, and, formerly, R. Migliorini and M. Pelagatti, are with Politecnico di Milano, Dipartimento di Elettronica e Informazione, Piazza L. da Vinci 32, 20133 Milano, Italy \{bascetta, magnani, rocco\}@elet.polimi.it
Systems of the first type, i.e. mounted above or near the robot, are dominating in industrial applications and research prototypes. They are usually based on non-contact measurement devices, like vision systems, Time Of Flight (TOF) cameras and laser scanners. Despite their wide use, however, these systems are strongly affected by occlusion effects, due to the mounting position of the measurement device, and do not allow for a close and safe human-robot interaction.

On the other hand, systems based on sensors mounted on the robot itself, usually based on contact (like force/torque) and non-contact (like ultrasonic or capacitive) measurement devices, are intrinsically exempt from occlusions. The performance of capacitive and ultrasonic devices, however, are strongly related to the environment conditions (e.g. material properties) and thus are not suitable to guarantee a constant and satisfactory safety level. These drawbacks can be overcome adopting optical devices, like TOF laser sensors.

TOF sensors are based on the measurement of the time needed for an emitted signal to be received (by reflection) by the emitting station. Physical principle might be ultrasound, radar, and optical, like laser sensors. Industrial applications of such sensors include measurement of levels in silos or containers, profile scanning, anti-collision systems in the automotive industry, traffic control. In the robotic area, an application of TOF sensors in mobile robotics is reported in [11]. Use of TOF cameras (i.e. devices that combine the functionalities of a camera system with those of a TOF matrix of distance sensors) is described in [12], with reference to mobile robotics, and in [13] with reference to a system designed for safe interaction between humans and robotic manipulators.

The present paper contributes proposing anti-collision methods based on the use of laser TOF as distance sensors. A protective barrier, referred to as virtual wall, is built around an obstacle, and is able to return a virtual force based on the sensed distance. Methods that preserve the spatial properties of a trajectory (GCT: Geometry Consistent Trajectory) in the presence of an obstacle, by suitably modifying the time evolution of the trajectory based on the sensed virtual force, are proposed, as well as methods that preserve the temporal properties of a trajectory (TCT: Time Consistent Trajectory), by modifying the reference signal based on an admittance control [14] on the sensed virtual force. The former method is a simplified version of [15] and accounts also for situations where the robot is moving away from a perceived obstacle.

Both the methods are experimentally evaluated on a testbed composed of a two-head linear motor equipped with 
a commercial laser TOF sensor.

Extensions from a 1D setting to a planar 2-d.o.f. manipulator equipped with a multiple beam laser TOF sensor is then proposed and discussed with respect to some theoretical properties. Validation through simulations is presented, that emphasize the different approaches of GCT and TCT methods.

\section{VIRTUAL WALL}

The anti-collision system based on the laser TOF sensor has been mainly developed in a 1D setting. Extension to a $2 \mathrm{D}$ setting will be presented in Section VI. The core of the method is the definition of a "virtual wall" i.e. a protective area around an obstacle where the robot senses artificial (virtual) repulsive forces, which tend to move it away from the obstacle. Fig. 1 shows the interaction between the robot and an obstacle placed along its rectilinear trajectory. In this scheme, $d$ denotes the measured (for instance through the laser TOF sensor) distance, $c$ the thickness of the virtual wall, $\mu=c-d$ the penetration (if positive) of the robot inside the virtual wall, $F_{w}$ the resulting virtual force returned by the wall.

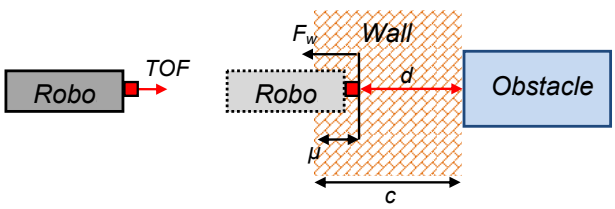

Fig. 1. Interaction between the robot and the virtual wall

The virtual wall can be attributed elastic as well as dissipative properties, which means that $F_{w}$ can be computed as:

$$
F_{w}= \begin{cases}K_{w} \mu+D_{w} \dot{\mu} & \text { if } \mu>0 \\ 0 & \text { if } \mu<0\end{cases}
$$

Let $M$ be the effective mass of the robot in motion, $d_{\text {min }}$ a minimum allowed distance between the robot and the obstacle, and $F_{\max }$ the maximum force the actuator of the robot can deliver. The gains $K_{w}$ and $D_{w}$ of the virtual wall can be selected assuming that when the minimum distance $d_{\min }$ is reached, the virtual wall applies the maximum force $F_{\max }$, and that the mass-spring-damper system formed by the robot and the virtual wall has a critical damping:

$$
K_{w}=\frac{F_{\max }}{c-d_{\min }}, \quad D_{w}=\sqrt{4 M K_{w}}
$$

\section{Geometry CONSISTEnt Trajectory}

A first way to use the computed value of the virtual force $F_{w}$, in order to avoid impacts between the robot and the obstacle, is to establish a force-dependent trajectory. This means that the trajectory evolves based on the virtual force, and specifically that the time variable used to define the said trajectory is updated based on this force. Let $x_{r}=x_{r}(t)$ be the expression of the trajectory the robot is expected to follow in an interval $T$. In the absence of any virtual force, the time index $t$ is updated according to the law $t_{k+1}=$ $t_{k}+\Delta t$, where $\Delta t$ is the (constant) time step in the trajectory generation. Similar to [15], the idea is to modify the update mechanism of the time variable based on the virtual force:

$$
\tau_{k+1}=\tau_{k}+\Delta t \cdot h\left(F_{w k}\right)
$$

where $\tau$ is the new force-dependent time index, $F_{w k}$ is the computed value of the virtual force at the $k_{t h}$ computation of the trajectory, while $h$ is the related corrective factor. Basically, the trajectory should be slowed down in case a virtual force from the obstacle is perceived, and actually stopped in case this force reaches a given threshold. In case this threshold is exceeded, the trajectory can be rewound, meaning that the time index is updated backwards (i.e. with a negative corrective factor). The corrective factor $h$ in (3) can thus be computed as follows:

$$
h\left(F_{w_{k}}\right): \begin{cases}=1 & \text { if } F_{w_{k}}=0 \\ \in(0,1) & \text { if } 0<F_{w_{k}}<F_{\text {stop }} \\ =0 & \text { if } F_{w_{k}}=F_{\text {stop }} \\ <0 & \text { if } F_{w_{k}}>F_{\text {stop }}\end{cases}
$$

The threshold $F_{\text {stop }}$ can be selected as one half of the maximum allowable force $F_{\max }$ defined in Section II. Furthermore a linear dependence of the corrective factor $h$ on the virtual force $F_{w}$ can be assumed, which means that (4) can be simplified as:

$$
h\left(F_{w_{k}}\right)=1-\frac{F_{w_{k}}}{F_{\text {stop }}}
$$

Notice that the above equation assumes that the trajectory forces the robot to move towards the obstacle. In case the robot perceives the obstacle (via the virtual force) while getting away from it, there is obviously no need to alter the trajectory generation. The two situations can be easily discriminated by checking the sign of the penetration velocity $\dot{\mu}$. Moreover the values of $h$ in (5) may need to be saturated when $F_{w_{k}}>F_{\text {stop }}$, in order to avoid excessively rapid variations in the reference trajectory.

Summarizing, equations (5) and (1) can be rewritten as follows:

$$
h\left(F_{w_{k}}\right)=\max \left(1-\frac{F_{w_{k}}}{F_{s t o p}}, h_{\text {min }}\right)
$$

where $h_{\min }<0$ is the minimum time decrement value and

$$
F_{w}= \begin{cases}K_{w} \mu+D_{w} \dot{\mu} & \text { if } \mu>0 \text { and } \dot{\mu}>0 \\ 0 & \text { otherwise }\end{cases}
$$

Fig. 2 shows an example of trajectory (from 0 to $2 \mathrm{~m}$ and backwards) when an obstacle is placed along the path. Notice that shortly after the trajectory enters the virtual wall, the evolution of the trajectory is stopped. It is then resumed when the obstacle is removed, with exactly the same time evolution as with the original trajectory $(h=1$ when there is no obstacle). This fact motivates the acronym GCT (Geometry Consistent Trajectory) that has been given to this anti-collision system, since the geometrical properties of the trajectory are preserved (whilst the time properties are released: the time to complete the trajectory depends on 


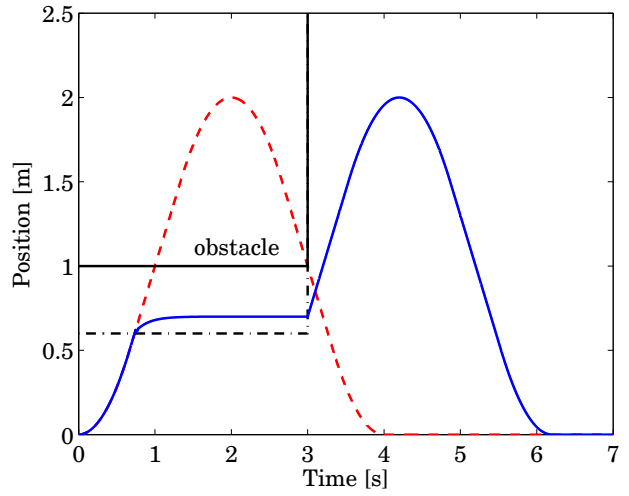

Fig. 2. Example of GCT. Dashed line: original trajectory; dashdot line: virtual wall; solid line: modified trajectory

the presence of obstacles along the path). Fig. 3 reports a conceptual block diagram of a GCT control system.

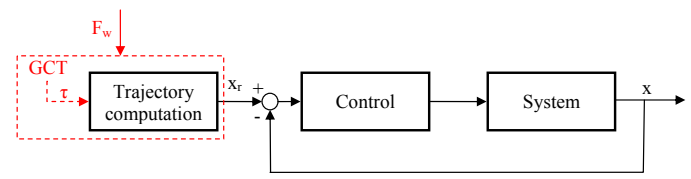

Fig. 3. Block diagram of a GCT system

\section{TIME CONSISTEnt TRAJECTORY}

A different way to use the virtual force in an anticollision system is to enforce a desired dynamic behaviour of the robotic system in response to this force. This can be accomplished through the well known tools of the admittance/impedance control [16], [17], [14]. For this, consider the block diagram sketched in Fig. 4, where $x_{r}$ is a reference trajectory, $x$ is the actual robot position, $d$ is the sensed distance from an obstacle, $F_{w}$ is the force delivered by the virtual wall, $G_{d}$ is an admittance filter. Assuming that the bandwidth of the motion control system is large enough to neglect its dynamic effects, a simple inspection of the block diagram yields:

$$
x \simeq x_{r}-G_{d}(s) F_{w}
$$

or:

$$
F_{w} \simeq G_{d}(s)^{-1}\left(x_{r}-x\right)
$$

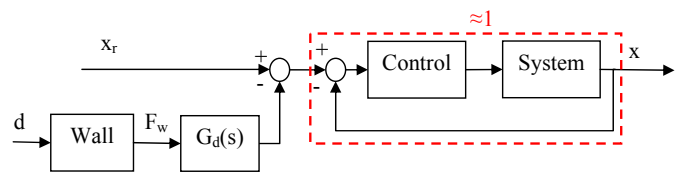

Fig. 4. Block diagram of an admittance controller fed by the virtual force

Thus the dynamics expressed by the admittance filter $G_{d}$ is imposed between position error and virtual force. Letting:

$$
G_{d}(s)=\frac{1}{M_{d} s^{2}+D_{d} s+K_{d}}
$$

the dynamics of a typical mass-spring-damper are assigned. The desired mass $M_{d}$ can be set equal to the actual mass $M$ of the robot (no mass scaling is needed), while the damping $D_{d}$ can be set so as to assign critical damping to the dynamics of the filter:

$$
D_{d}=\sqrt{4 K_{d} M}
$$

Notice that the distance $d$ in Fig. 4 obviously depends on the position itself of the robot, which implies that a further loop is closed on the system. This is shown in Fig. 5, where $x_{o}$ is the position of the obstacle. It is straightforward to derive the characteristic polynomial of this closed loop system as:

$$
M_{d} s^{2}+\left(D_{d}+D_{w}\right) s+K_{d}+K_{m}
$$

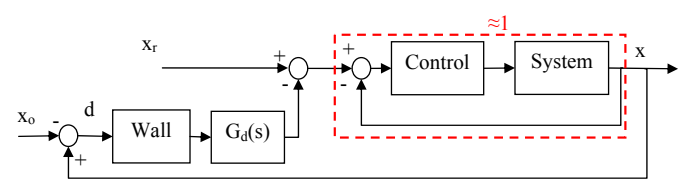

Fig. 5. Block diagram of the admittance controller with the explicit computation of the distance $d$

The damping factor of the roots of this polynomial is:

$$
\zeta=\frac{1}{2} \frac{D_{d}+D_{w}}{\sqrt{\left(K_{d}+K_{w}\right) M_{d}}}
$$

Selecting the damping coefficients $D_{w}$ and $D_{d}$ as in (2) and (11), respectively, and taking $M_{d}=M$, it turns out that:

$$
\zeta=\frac{\sqrt{K_{d}}+\sqrt{K_{w}}}{\sqrt{\left(K_{d}+K_{w}\right)}}>1
$$

Thus the closed loop system has always real eigenvalues when the parameters of the virtual wall and of the admittance filter are selected as described.

Guidelines for the selection of the parameter $K_{d}$ can be derived making reference to the conceptual representation in Fig. 6. The representation shows that when the robot is tracking a reference, and an obstacle appears along the way, the virtual wall and the impedance filter act as contrasting springs. A large value of $K_{d}$ implies a stiff reaction of the robot to changes in the position reference and consequently a risk of impact with the obstacle. On the other hand, small values of $K_{d}$ might yield a sluggish trajectory tracking when the obstacle disappears. A workaround might be to replace the linear relation expressed by the spring with stiffness $K_{d}$ with a nonlinear function that saturates the force delivered by the admittance filter to a maximum desired value $F_{e q}$, when the error between the reference and actual position reaches a limit value $\Delta x_{l i n} . F_{e q}$ can be computed assuming an equilibrium with the force delivered by the virtual wall:

$$
F_{e q}=K_{w}\left(c-d_{e q}\right)
$$

where $d_{e q}$ is the desired robot-obstacle distance at the equilibrium point, while the maximum value of the error 


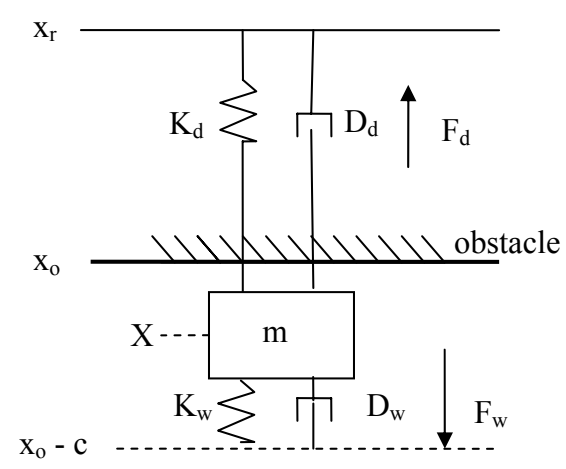

Fig. 6. Conceptual representation of the actions of the virtual wall and of the admittance filter on the robot

for a linear behaviour of the spring, $\Delta x_{l i n}$, can be selected equal to the thickness $c$ of the virtual wall.

Fig. 7 shows an example of trajectory when an obstacle is placed along the path. Notice that the modified trajectory does not reach the final point, and in the final part the original trajectory is resumed. The time properties of the trajectories are then preserved, while the geometrical properties are released. This fact motivates the acronym TCT (Time Consistent Trajectory) given to this anti-collision system.

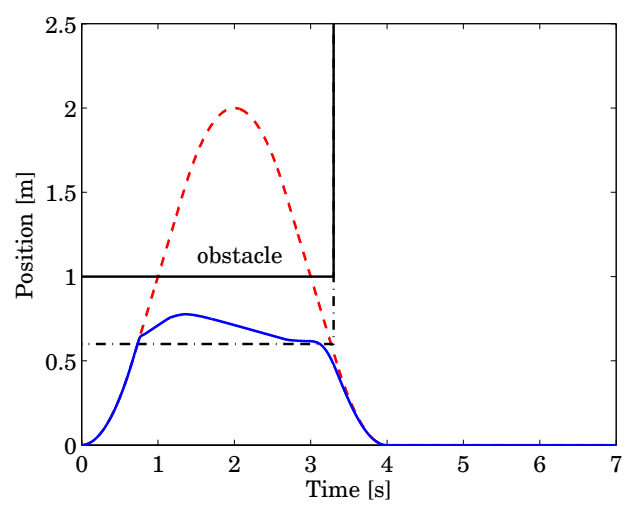

Fig. 7. Example of TCT. Dashed line: original trajectory; dashdot line: virtual wall; solid line: modified trajectory

\section{EXPERIMENTAL RESULTS}

The GCT and TCT methods have been tested on a prototype system based on a linear motor and shown in Fig. 8. The system is composed of a rectilinear cast-iron basement over which two translating tables (the heads) slide along the same axis. The overall extension of the system is about $2.8 \mathrm{~m}$. The permanent magnet synchronous linear motors (SKA.55.HS.275.14) are manufactured by Motor Power Company. Linear encoders are used to measure the positions of both the sliders. The machine is equipped with servodrives (Unidrive SP) manufactured by Control Techniques. In this application, one out of the two heads of the linear motor plays the role of the robot, whose task is to track a given trajectory avoiding impacts with the other head or any other

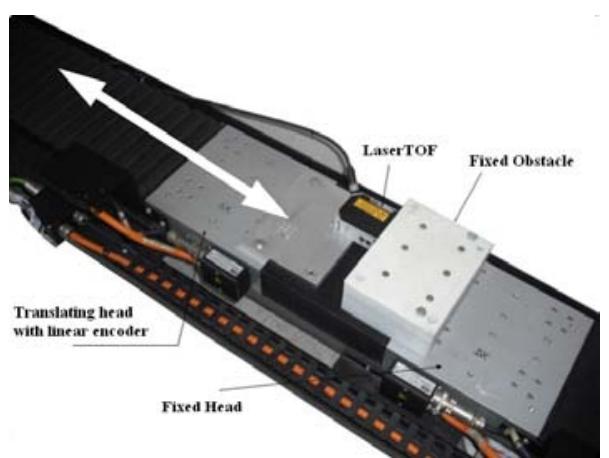

Fig. 8. Experimental setup

obstacle. For this, a commercial laser TOF sensor has been mounted on top of the first head: the sensor (BOD 63MLA02-S115) is manufactured by Balluff. It has a working range from $200 \mathrm{~mm}$ to $2 \mathrm{~m}$, nominal response time less than $2 \mathrm{~ms}$, nominal resolution less than $1 \mathrm{~mm}$.

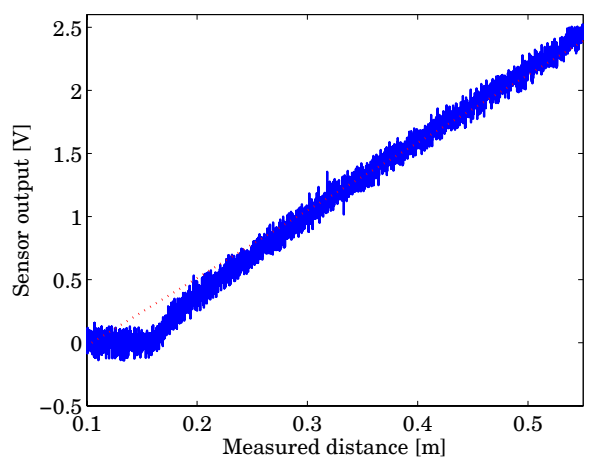

Fig. 9. Laser TOF sensor experimental characteristic

First experiments have been carried out in order to characterize the laser TOF distance sensor. Starting from the configuration reported in Fig. 8, where the lens of the sensor makes contact with the obstacle placed on one end of the rail, several synchronous acquisitions of the TOF sensor and of the linear encoder have been obtained during motion of the head carrying the sensor. The outcome of one of these experiments is reported in Fig. 9: it is shown that the sensor is unable to perceive objects less than about $16 \mathrm{~cm}$ distant, and that it exhibits a linear characteristic between distance and related measurement beginning with distances larger than $20 \mathrm{~cm}$. These results are consistent with the sensor nominal features. Fig. 10 shows a step response of the sensor, obtained using an obstacle that suddenly appears or disappears in front of the laser. Sampling time was $250 \mu \mathrm{s}$, which means that more than $60 \%$ of the overall variation of the sensor output is reached within $2 \mathrm{~ms}$, again consistent with the product data sheet. Notice the nonlinear behaviour of the time response of the sensor.

Once the sensor has been characterized, the whole experimental setup has been used to test the GCT and TCT anticollision systems. A virtual wall with a thickness $c=0.7 \mathrm{~m}$ and a minimum allowed distance $d_{\min }=0.25 \mathrm{~m}$ has been 


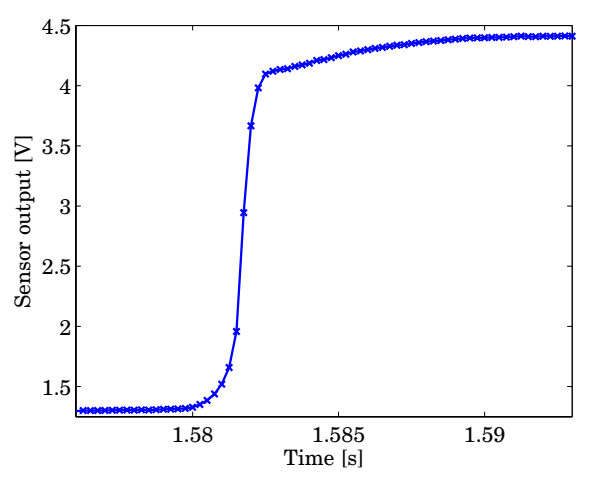

Fig. 10. Laser TOF sensor experimental response time

adopted, while the equilibrium distance $d_{e q}$ used in the TCT method (15) has been set to $0.25 \mathrm{~m}$. A reference trajectory characterized by a trapezoidal velocity profile, with an overall displacement of $1.3 \mathrm{~m}$ in $2 \mathrm{~s}$ has been programmed $^{1}$ and executed forward and backwards.

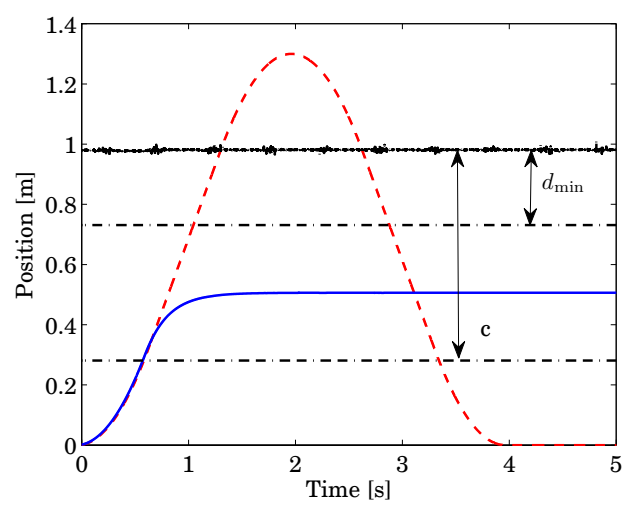

Fig. 11. Fixed obstacle: GCT method. Dashed line: original trajectory; dashdot line: virtual wall; solid line: modified trajectory

Figs. 11 and 12 report the results when a fixed obstacle is placed along the planned trajectory, using a GCT and a TCT system, respectively. Notice that with the GCT system the head settles in a position inside the virtual wall, with a distance from the obstacle larger than the minimum allowed one $d_{\text {min }}$. On the other hand, with the TCT system the head returns to the home position in the specified time, as expected.

Then the situation when a fixed obstacle suddenly vanishes has been tested. Fig. 13 shows the performance of the GCT system. Notice that the trajectory is correctly executed, once the obstacle has disappeared. On the other hand, the TCT method (see Fig. 14, where only the forward part of the trajectory has been assigned) shows that when the obstacle disappears, the trajectory exhibits the dynamics of a second order filter (the admittance filter) fed by a step equal to the difference between the original value of the trajectory and the

\footnotetext{
${ }^{1}$ The maximum velocity and acceleration amount to $0.975 \mathrm{~m} / \mathrm{s}$ and $1.463 \mathrm{~m} / \mathrm{s}^{2}$, respectively.
}

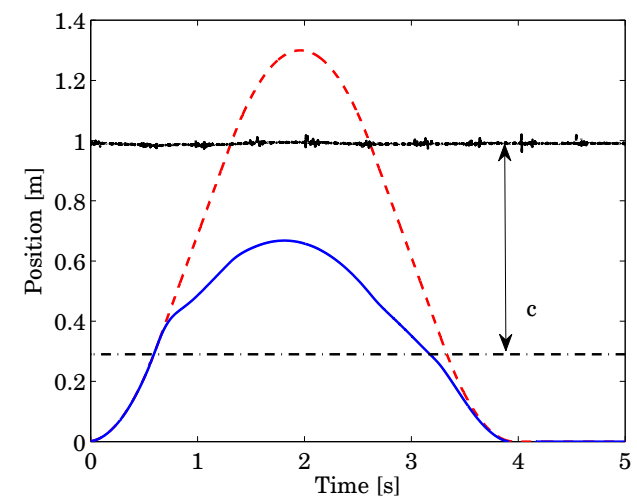

Fig. 12. Fixed obstacle: TCT method. Dashed line: original trajectory; dashdot line: virtual wall; solid line: modified trajectory

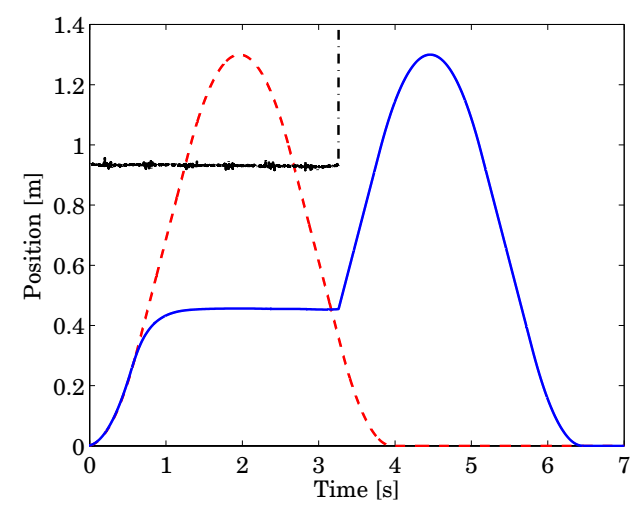

Fig. 13. Vanishing obstacle: GCT method. Dashed line: original trajectory; dashdot line: virtual wall; solid line: modified trajectory

current position. As a matter of fact the reaction is limited by the saturation function described in Section IV.

\section{EXTENSION TO A PLANAR MANIPULATOR}

The anti-collision systems described in the previous Section can be extended to cope with more challenging situations than the 1D scenario adopted so far. In the following, the case of a planar manipulator will be briefly discussed. Reference is made to a 2 d.o.f. manipulator with rotational joints, where a system composed of a combination of laser sensors is mounted at the end-effector (see Fig. 15). Each sensor measures a distance from an obstacle along its ray. The vector forces are then added together so as to give the total virtual force acting at the end-effector. A certain number $n_{T O F}$ of sensors should be adopted: this number can be related to the minimum length of the part of the obstacles in front of the sensors, $\Phi_{\min }$, and to the desired thickness of the virtual wall $c$ by the following relation:

$$
\frac{2 \pi c}{\Phi_{\min }}=n_{T O F}
$$

as it is easily derived from Fig. 16. Note that if (16) is satisfied, the discrete nature of the sensor does not lead to jumps in the virtual force. 


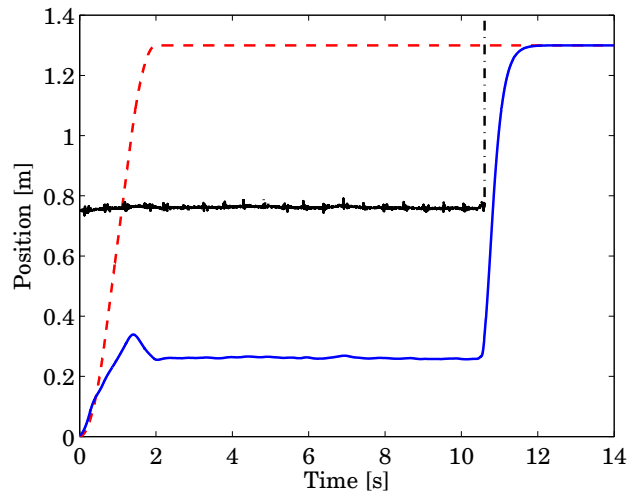

Fig. 14. Vanishing obstacle: TCT method. Dashed line: original trajectory; dashdot line: virtual wall; solid line: modified trajectory

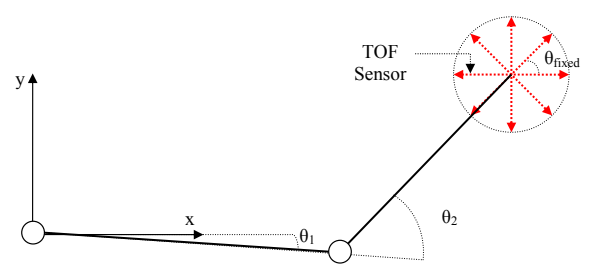

Fig. 15. Planar robot manipulator with anti-collision sensors at the endeffector

Both a GCT and a TCT system have been implemented, in simulation. In the GCT system, a geometrical path for the end-effector is first assigned and then the time evolution of the trajectory is conditioned to the experienced virtual force in a similar fashion as in (3). On the other hand, in the TCT solution the trajectories along the $x$ and $y$ coordinates are independently assigned and modified based on the $x$ and $y$ components of the virtual force at the end-effector, respectively. This way the anti-collision system is allowed to search alternative geometrical paths in order to complete the trajectory in a consistent time fashion. The block diagram of this TCT system extends the scheme already reported in Fig. 5 for the 1D situation, and is sketched in Fig. 17. Assuming large enough bandwidths of the motion control loops, and an admittance filter $G_{d}(s)$ defined as in (10), it is straightforward to verify that the dynamics of the closed loop system are described by the following time-domain equation:

$$
M_{d} \ddot{e}+D_{d} \dot{e}+\left(K_{d} I+W\right) e=W z_{d}
$$

where $z=(x, y), z_{d}=\left(x_{d}, y_{d}\right), e=z_{d}-z$ and $W$ is the matrix relation imposed by the virtual wall between position

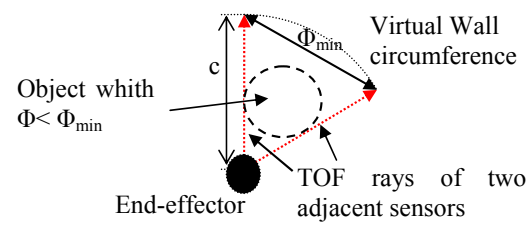

Fig. 16. Computation of the minimum number of sensors

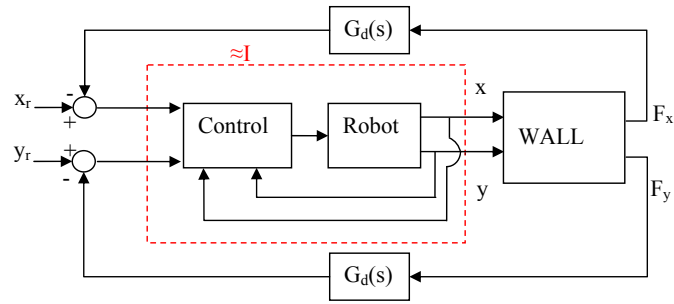

Fig. 17. Block diagram of the TCT system in a 2D situation

and virtual force. Consider first a single laser sensor, as depicted in Fig. 18. Simple geometrical considerations lead to the following expression of matrix $W$ :

$$
W=K_{m}\left[\begin{array}{cc}
-\frac{m_{M}}{m-m_{M}} & \frac{1}{m-m_{M}} \\
-\frac{m_{M} m}{m-m_{M}} & \frac{m}{m-m_{M}}
\end{array}\right]
$$

where $K_{m}$ is the scalar stiffness of the virtual wall, while $m$ and $m_{M}$ are slopes of the lines defining the ray of incidence of the sensor and the plane of the obstacle, respectively (see Fig. 18). In case a continuous (in space) sensor is mounted at the end-effector (see Fig. 19) it can be proven that the resulting virtual force $F_{t o t}$ is orthogonal to the face of the obstacle, i.e. $m \cdot m_{M}=-1$. In this case, matrix $W$ takes the following expression:

$$
W=K_{m} \frac{1}{1+m_{M}^{2}}\left[\begin{array}{cc}
m_{M}^{2} & -m_{M} \\
-m_{M} & 1
\end{array}\right]
$$

This matrix is positive semi-definite: thus it establishes a physically consistent elastic relation between position coordinates $x$ and $y$ and force coordinates $F_{x}$ and $F_{y}$. Once plugged into (17) it yields an asymptotically stable closedloop system, provided that $M_{d}, D_{d}$ and $K_{d}$ are positive gains. In case a discrete sensor is used, this result is obviously valid only asymptotically, at increasing number of sensors.

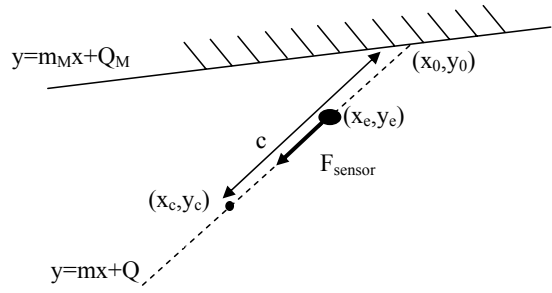

Fig. 18. Single sensor interacting with an obstacle

Simulations have been run on the model of a 2 d.o.f. manipulator with a sensor composed of a combination of eight equally distributed 1D laser TOF sensors (Fig. 15). A moving obstacle has been introduced along the path to be followed by the end-effector. Figs. 20 and 21 show the results of the simulations, for the GCT and the TCT systems, respectively. Notice that with the GCT system the obstacle is avoided while preserving the geometrical path, while with the TCT system the final point is reached in the prescribed 


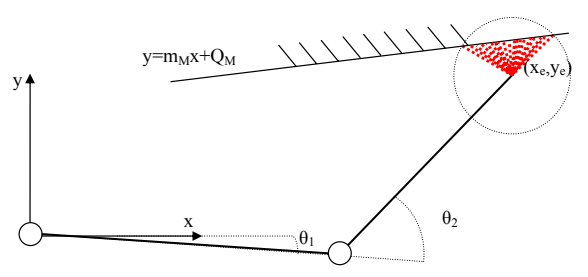

Fig. 19. Continuous sensor interacting with an obstacle

time, avoiding the obstacle through a different geometrical path.
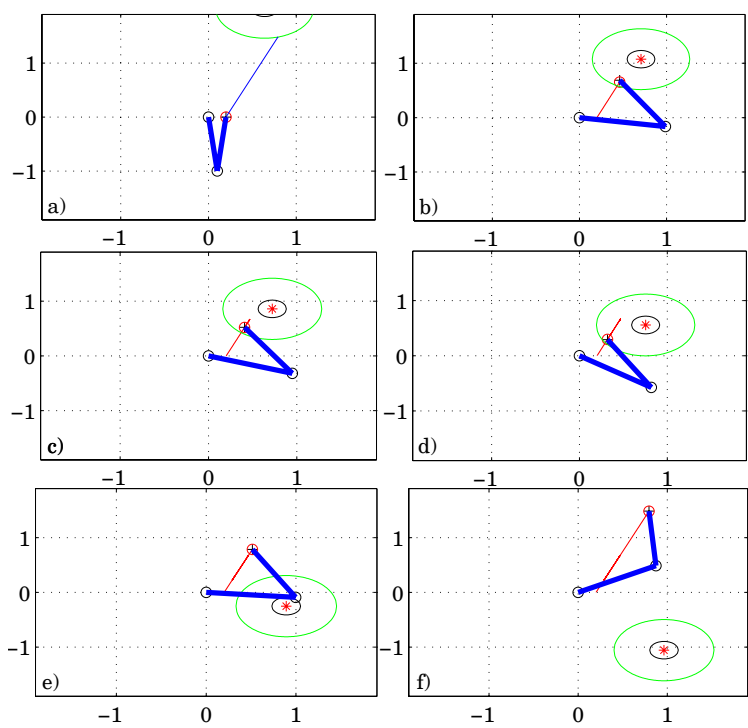

Fig. 20. Simulation of a GCT system with a moving obstacle

\section{CONCLUSIONS}

Anti-collision strategies have been discussed in this paper. Laser TOF sensor has proven to be a suitable device (in terms of resolution and response time) to allow for effective human-robot interaction control. The virtual force that is formed based on the sensed distance can be used in different ways to modify the trajectory, either preserving its geometrical properties or its temporal properties. Experimental validation on a $1 \mathrm{D}$ setting has been documented in the paper while validation in a $2 \mathrm{D}$ scenario is an ongoing work.

\section{ACKNOWLEDGEMENTS}

The research leading to these results has received funding from the European Community's Seventh Framework Programme FP7/2007-2013 - Challenge 2 - Cognitive Systems, Interaction, Robotics - under grant agreement No. 230902ROSETTA. This document reflects only the authors views and the European Community is not liable for any use that may be made of the information contained herein.

\section{REFERENCES}

[1] A. De Santis, B. Siciliano, A. De Luca, and A. Bicchi, "An atlas of physical human-robot interaction," Mechanism and Machine Theory, vol. 43 , pp. $253-270,2008$.
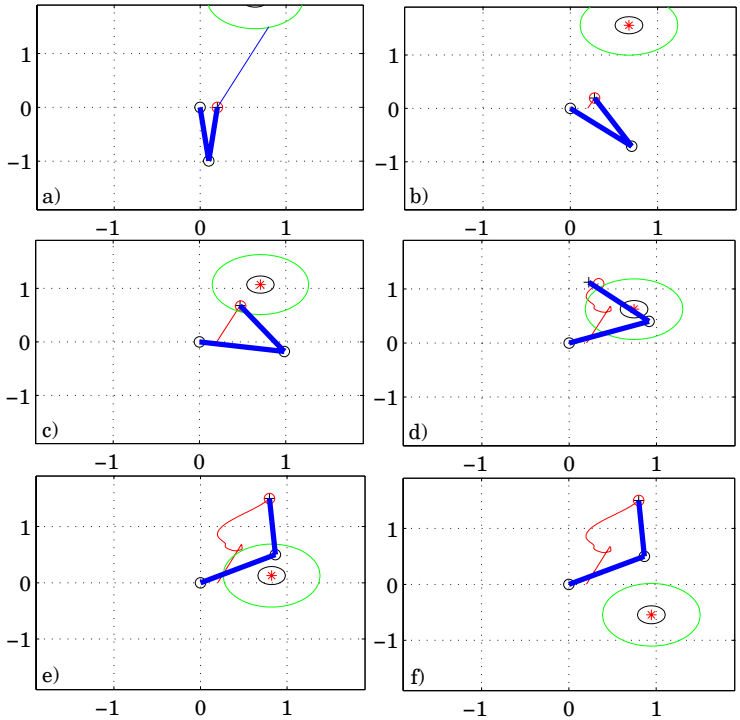

Fig. 21. Simulation of a TCT system with a moving obstacle

[2] A. Bicchi and G. Tonietti, "Fast and soft arm tactics - dealing with the safety-performance trade-off in robot arms design and control," IEEE Robot. Automat. Mag., vol. 11, pp. 22-33, 2004.

[3] S. Haddadin, A. Albu-Schäffer, and G. Hirzinger, "Safety evaluation of physical human-robot interaction via crash-testing," in Proc. Robotics Science and Syst. Conf., 2007.

[4] S. Oberer and R. Schraft, "Robot-dummy crash tests for robot safety assessment," in Proc. IEEE Rob. Autom. Conf., 2007, pp. 2934-2939.

[5] M. Zinn, O. Khatib, B. Roth, and J. Salisbury, "Playing it safe - humanfriendly robots," IEEE Robot. Automat. Mag., vol. 11, pp. 12-21, 2002.

[6] K. Ikuta, M. Nokata, and H. Ishii, "Safety evaluation method of design and control for human-care robots," Int. J. Robot. Res., vol. 22, pp. 281-297, 2003.

[7] J. Heinzmann and A. Zelinsky, "Quantitative safety guarantees for physical human-robot interaction," Int. J. Robot. Res., vol. 22, pp. 479-504, 2003.

[8] D. Kulic and E. Croft, "Real-time safety for human-robot interaction," Robotic Autonomous Systems, vol. 54, pp. 1-12, 2006.

[9] A. Bicchi, M. Peshkin, and E. Colgate, "Safety for physical humanrobot interaction," in Handbook of Robotics. Springer, 2008.

[10] A. Pervez and J. Ryu, "Safe physical human robot interaction - past, present and future," J. Mech. Sci. and Technol., vol. 22, pp. 469-483, 2008.

[11] U. Larsson, J. Forsberg, and A. Wernersson, "Mobile robot localization: integrating measurements from a time-of-flight laser," IEEE Trans. Ind. Electron., vol. 43, pp. 422-431, 1996.

[12] S. May, B. Werner, H. Surmann, and K. Pervölz, "3D time-of-flight cameras for mobile robotics," in Proc. IEEE/RSJ Int. Conf. Intell. Robot Systems, 2006, pp. 790-795.

[13] B. Winkler, "Safe space sharing human-robot cooperation using a 3D time-of-flight camera," in Proc. Int. Robots Vision Show, ISR 2007, 2007

[14] G. Ferretti, G. Magnani, and P. Rocco, "Assigning virtual tool dynamics to an industrial robot through an admittance controller," in Proc. IEEE Int. Conf. Adv. Robot., 2009.

[15] S. Haddadin, A. Albu-Schäffer, A. De Luca, and G. Hirzinger, "Collision detection and reaction: a contribution to safe physical humanrobot interaction," in Proc. IEEE/RSJ Int. Conf. Intell. Robot Systems, 2008, pp. 3356-3363.

[16] N. Hogan, "Impedance control: an approach to manipulation. part Theory," ASME J. of Dyn. Syst., Meas., and Contr., vol. 107, pp. 1-24, 1985.

[17] G. Ferretti, G. Magnani, and P. Rocco, "Impedance control for elastic joints industrial manipulators," IEEE Trans. Robot., vol. 20, pp. 488 498, 2004. 